\title{
Highlights in purinergic signaling
}

\author{
Nicholas Dale
}

Published online: 31 October 2009

(C) Springer Science + Business Media B.V. 2009

Nicke A, Kuan YH, Masin M, Rettinger J, MarquezKlaka B, Bender O, Górecki DC, Murrell-Lagnado RD, Soto F (2009) A functional P2X7 splice variant with an alternative transmembrane domain 1 escapes gene inactivation in $\mathbf{P 2 X} 7 \mathrm{KO}$ mice. J Biol Chem: doi: 10.1074/jbc.M109.033134

\section{Article summary}

The authors describe a new version of the $\mathrm{P} 2 \mathrm{X} 7$ receptor, P2X7k, which starts at an alternative transcription initiation site in what was thought to be a large intron between the first and second exons. This provides a functional receptor with an alternative first transmembrane domain. There are three key findings of this work: (1) the P2X7k receptor has distinct signaling properties and notably undergoes pore dilation very rapidly; (2) it strengthens the case that pore dilation is a property of the $\mathrm{P} 2 \mathrm{X} 7$ protein rather than additional proteins; and (3) in transgenic mice where gene silencing of the P2X7 receptor has been attempted by removal of the first exon, $\mathrm{P} 2 \mathrm{X} 7 \mathrm{k}$ is still expressed and is functional.

Peng W, Cotrina ML, Han X, Yu H, Bekar L, Blum L, Takano T, Tian GF, Goldman SA, Nedergaard M. (2009) Systemic administration of an antagonist of the ATPsensitive receptor $\mathrm{P} 2 \mathrm{X} 7$ improves recovery after spinal cord injury. Proc Nat Acad Sci USA 106, 12489-12493.

\section{Article summary}

One of the major problems with spinal cord injury is that secondary scarring following the initial lesion prevents

\footnotetext{
N. Dale $(\bowtie)$

Department of Biological Sciences, University of Warwick, Coventry CV4 7AL, UK

e-mail: n.e.dale@warwick.ac.uk
}

reestablishment of neural connections and thus results in permanent paralysis. Prior evidence suggested that massive release of ATP following injury could, by acting via P2X7 receptors, trigger the cellular processes leading to inflammation and scarring. The authors set out to investigate a new therapeutic approach in a rat spinal cord injury model by testing whether an antagonist of $\mathrm{P} 2 \mathrm{X} 7$ receptors, Brilliant Blue $\mathrm{G}$-closely related to a food coloring agent and which can cross the blood-brain barrier, could prevent the activation of astrocytes and microglia and thus reduce scarring. Remarkably, this approach reduced the secondary scarring and improved the restoration of motor behavior.

\section{Combined commentary}

ATP can act at ligand-gated channels, known as P2X receptors of which there are seven subtypes. Some of these subtypes can combine to form heteromeric channels but others form only homomeric channels. The P2X7 receptor is thought to fall into this latter category, although a recent report suggests that in heterologous expression systems P2X4 and P2X7 receptors may indeed form functionally distinct heteromeric channels (Guo C., et al., Mol Pharmacol (2007) 72: 1447-56). The P2X7 receptor has other features that make it somewhat different from the other P2X receptors: while sharing the same overall topology with other P2X subunits (two transmembrane segments and a large extracellular loop) it has a very large intracellular C terminal domain; it has relatively low affinity for ATP; and was described as the first $\mathrm{P} 2 \mathrm{X}$ receptor to undergo poredilation during continued exposure to ATP (Surprenant A. et al., Science (1996) 272: 735-738) - a high conductance state that allows passage of molecules of up to a few hundred daltons. Subsequently, pore dilation has been shown to occur for some other P2X receptors (Khakh B. S. et al., Nat Neurosci (1999) 2: 322-330). The mecha- 
nisms of pore dilation are controversial: some authors contend that accessory proteins, namely pannexin-1, are required (Pelegrin P. and Surprenant A., EMBO J (2006) 25: 5071-5082); while others posit that it is an intrinsic property of the channel itself (e.g. Yan Z. et al., J Gen Physiol (2008) 132: 563-573). Nevertheless, P2X7 receptors have attracted considerable interest as they have been characterized as "death channels" owing to their ability to undergo pore dilation, permeabilize cells, and ultimately trigger apoptosis.

Prior to this work, the $\mathrm{P} 2 \mathrm{X} 7$ receptor gene was described as having 13 exons distributed over more than $40 \mathrm{kB}$ of DNA. Previous work had documented nine alternative splice variants (Cheewatrakoolpong et al. 2005, Biochem Biophys Res Commun 332: 17-27). However, only one of these (with a truncated C-terminal cytoplasmic domain), is capable of forming a functional channel. Nicke et al. have found an alternative transcriptional start and first exon (exon $1^{\prime}$ ) downstream from exon 1 in what was previously thought to be an intron. This alternative transcription start gives rise to a receptor termed P2X7k (the first version of the P2X7 receptor comprised of exons 1-13, but not exon $1^{\prime}$, is known as $\mathrm{P} 2 \mathrm{X} 7 \mathrm{a}$ ). Could this mean that there are potentially several different splice variants of the $\mathrm{P} 2 \mathrm{X} 7 \mathrm{k}$ channel analogous to those described for $\mathrm{P} 2 \mathrm{X} 7 \mathrm{a}$ ?

$\mathrm{P} 2 \mathrm{X} 7 \mathrm{k}$ forms a functional channel and is expressed in many tissues including brain, spinal cord, kidney, liver, thymus, spleen, and heart. The properties of P2X7k are significantly different from those of P2X7a. Firstly, the P2X7k has a higher affinity for the agonist Bz-ATP (eightfold higher than P2X7a). Secondly, the P2X7k enters into the dilated pore state very rapidly compared to the P2X7a, which requires a longer exposure to a higher concentration of ATP. In some ways, therefore, activation of $\mathrm{P} 2 \mathrm{X} 7 \mathrm{k}$ is potentially a more powerful and sensitive trigger for cell death. This last finding is also convergent with the prior demonstration that mutations of the $\mathrm{P} 2 \mathrm{X} 7$ gene can alter the ability of the channel to enter into the dilated state (Yan Z. et al., J Gen Physiol (2008) 132: 563-573). This is most simply explained by positing that pore dilation is an intrinsic function of the channel rather than requiring the involvement of another protein.

This paper conveys a powerful message for all investigators seeking to delete genes and assay function. In one transgenic mouse, the P2X7 gene was "deleted" by inserting a lacZ reporter gene only into the first exon. This had the effect of deleting expression of P2X7a but, as Nicke et al. have elegantly shown, expression of P2X7k is unaffected in this strain of mouse. Thus, the design of constructs to delete expression of complex genes must carefully take into account both alternative splicing and alternative start sites for transcription. Another implication of this finding is that the distribution of $\mathrm{P} 2 \mathrm{X} 7$ receptors particularly in brain should be reassessed. Sim et al. (J Neurosci (2004) 24: 6307-6314) used the lacZ P2X7 knock-in mouse to conclude, on the basis of the lack of lacZ expression in neurons, that the $\mathrm{P} 2 \mathrm{X} 7$ receptor is not expressed in neurons. Yet, functional evidence (e.g., Leon D. et al., Eur J Neurosci (2006) 23: 2999-3013) suggests the possibility of neuronal $\mathrm{P} 2 \mathrm{X} 7$ receptors. This latest finding of an alternative transcription start site weakens the conclusion of Sim et al. and suggests a potential resolution of conflicting evidence, as the lacZ reporter in exon 1 utilized in this prior study would have demonstrated only the distribution of $\mathrm{P} 2 \mathrm{X} 7 \mathrm{a}$ and not that of $\mathrm{P} 2 \mathrm{X} 7 \mathrm{k}$ receptors. Thus, it would be well worth investigating whether P2X7k receptors (and potential splice variants thereof) could be expressed in neurons.

P2X7 receptors are highly expressed in spinal cord. Spinal cord injury can result in permanent paralysis as nerve fibers are unable to reconnect with their targets. The inability to reconnect seems to result from secondary tissue damage arising from inflammation and scarring. This creates a barrier that impedes correct axon regrowth and navigation. During traumatic injury, ATP will be released from damaged cells and reach rather high concentrations in the extracellular space. This will activate a variety of receptors including the $\mathrm{P} 2 \mathrm{X} 7$ receptor. Prior investigations had shown that antagonists of $\mathrm{P} 2 \mathrm{X} 7$ receptors could reduce inflammation and scarring occurring in response to spinal injury as well as promoting functional recovery. However, a useful therapeutic agent must be non-toxic and able to cross the blood-brain barrier. Thus, the realization of Peng et al. that a well-known P2X7 antagonist, BBG, is analogous to a very safe food dye was highly significant. Following their insight, Peng et al. demonstrated in a rat model of spinal cord injury that systemic application of BBG (intravenous infusion) given after the trauma was able to reduce the activation of astrocytes, microglia, and neutrophils around the injury site and enhance the recovery of motor function. As there are no particularly effective treatments for spinal injury, this is a discovery of immense practical importance and could lead fairly rapidly to applications in humans. To link the two papers that I have chosen on this topic -could this be a role of the $\mathrm{P} 2 \mathrm{X} 7 \mathrm{k}$ receptor? This question is easily answerable with the mouse in which only exon 1 has been deleted, leaving the $\mathrm{P} 2 \mathrm{X} 7 \mathrm{k}$ variant as the only known $\mathrm{P} 2 \mathrm{X} 7$ receptor.

\section{Halassa MM, Florian C, Fellin T, Munoz JR, Lee SY, Abel T, Haydon PG, Frank MG (2009) Astrocytic modulation of sleep homeostasis and cognitive consequences of sleep loss. Neuron 61, 213-219.}

\section{Article summary}

The authors investigate astrocytes as a possible source of adenosine that accumulates during wakefulness and 
mediates an increased pressure to sleep following prolonged wakefulness. By using a transgenic mouse (dnSNARE) with selective blockade of exocytosis only in astrocytes, the authors demonstrate a disturbance in sleep homeostasis and reduced recovery sleep following sleep deprivation. These effects can be respectively mimicked in wild-type mice and occluded in the dnSNARE mice by application of an A1 adenosine receptor antagonist. They then demonstrate that astrocyte-derived adenosine contributes to memory loss following sleep deprivation. Astrocytes are thus identified as important regulators of sleep and memory formation.

Bjorness TE, Kelly CL, Gao T, Poffenberger V, Greene RW (2009) Control and function of the homeostatic sleep response by adenosine A1 receptors. J Neurosci 29, 1267-1276.

\section{Article summary}

Synchronized slow wave activity across the CNS is a prominent feature of slow wave sleep. This slow wave activity directly correlates with the preceding duration of wakefulness and is enhanced by sleep deprivation. Nevertheless, the role of slow wave activity as opposed to sleep duration in maintenance of cognitive performance has not been established. The authors have used a conditional targeted knock-out strategy to reduce adenosine A1 receptor expression in all of the sleep relevant brain areas. While this genetic manipulation did not affect the duration of rebound sleep, it did affect rebound slow wave activity in response to sleep deprivation. The authors then probed the consequences of sleep deprivation for working memory in wild type and in mice with targeted deletion of the A1 receptor. Interestingly, the mice with targeted A1 receptor deletion showed deficits in working memory following sleep deprivation (but not under control conditions) suggesting that homeostatic control of slow wave activity rather than sleep duration per se is important for maintenance of cognitive function.

\section{Combined commentary}

Sleep not only exhibits a circadian rhythm but is also subject to homeostatic control-we tend to sleep a fixed amount each day and if for some reason we are unable to sleep, when sleep becomes possible we sleep for longerso called rebound or recovery sleep. Adenosine has long been known as an endogenous sleep-promoting substance or somnogen (Radulovacki M. et al., Brain Res (1983) 271: 392-395). The finding of Porkka-Heiskanen T. et al. (Science (1997) 276: 1265-1268) that levels of adenosine in the sleep promoting areas of the brain increase with prolonged wakefulness and that blockade of adenosine transport and the consequent increase in extracellular adenosine in the basal forebrain will increase slow wave sleep firmly established adenosine as playing a fundamental role in sleep homeostasis. These two highlighted papers address different aspects of adenosine's role.

The first, Halassa et al., seeks to understand where the adenosine comes from. These authors come up with a surprising answer-at least in part from astrocytes. These authors use a sophisticated genetically altered mouse in which a truncated synaptobrevin molecule (termed dnSNARE) which has dominant negative effects on SNARE-dependent vesicular fusion can be expressed conditionally only in astrocytes (Pascual O. et al., Science (2005) 310: 113-116). This manipulation thus selectively blocks vesicular fusion in a large proportion of astrocytes; once this is done, adenosine levels in the brain fall, as measured through A1 receptor antagonism. Pascual et al. explained this through reduced release of ATP from astrocytes and hence less substrate for ectoATPases and less production of adenosine in the extracellular space. This prior finding led Halassa and colleagues to ask whether the dnSNARE mice could have a sleep phenotype.

There are two major phases of sleep with very distinct characteristics: REM (rapid eye movement sleep-the phase in which we have exciting dreams, but are unable to act them out) and slow wave sleep. In slow wave sleep the brain is relatively less active than waking and the cortical EEG is characterized by synchronized slow waves or delta waves (over a frequency range of 0.5 to $4.5 \mathrm{~Hz}$ ). The power of these slow waves (delta power) is a measure of both the depth of sleep and the drive to sleep. The dnSNARE mice exhibited less delta power than their wild type littermates (particularly in the 0.5 to $1.5 \mathrm{~Hz}$ range) during the normal sleep-wake cycle. This effect was especially prominent following sleep deprivation and the dnSNARE mice exhibited a much shorter duration of rebound sleep following sleep deprivation than their wild type littermates. Why is this effect mediated by adenosine? Halassa and colleagues show that the A1 antagonist CPT applied via intracerebroventricular injection decreased total sleep time in the wild type but had no effect on this parameter in dnSNARE mice. Indeed application of CPT in the wild type replicates the dnSNARE phenotype. To complete an already landscape-changing study these authors then asked whether dnSNARE mice could have altered memory, as there are many indications that there is an important relationship between sleep and memory consolidation. To approach this, they utilized a novel object recognition test. The mice were trained on day 1 and either allowed uninterrupted sleep or were sleep-deprived for $6 \mathrm{~h}$ and retested the next day. Normally, mice will spend more time with a novel object and this was indeed found for wild type mice allowed an interrupted night's sleep. This preference for the novel object was reduced to chance 
levels by sleep deprivation in wild-type mice. The dnSNARE mice had a similar capacity to the wild-type mice to recognize a novel object. However, in the dnSNARE mice, unlike in their wild-type siblings, sleep deprivation did not diminish their ability to recognize a novel object. Once again, as CPT could replicate this phenotype, the authors propose that this is mediated by the altered accumulation of adenosine in the dnSNARE mice. This would suggest that normally during sleep deprivation increased adenosine accumulation prevents the consolidation of labile memories. There is some evidence that this is plausible from the work of Lynch and colleagues demonstrating that adenosine can prevent/erase LTP in the hippocampus during a labile period (Arai A. et al., Neurosci Lett (1990) 119: 41-44; Rex C.S. et al., J Cell Biol (2009) 186: 85-97). So, astrocytes go from "brain glue" to mediators of sleep homeostasis and modulators of memory consolidation via adenosine in one paper.

The second paper addresses the aspects of slow wave sleep that are important for its function. In particular is the duration of slow wave sleep or its quality as measured by the amount of slow wave activity (SWA) important? In the terminology used above, SWA was measured in a subset of the delta wave frequency range from 3 to $4.5 \mathrm{~Hz}$. The starting point for these authors was the puzzling and contradictory finding that constitutive deletion of the A1 receptor had no effect on sleep or rebound sleep (Stenberg D. et al., J Sleep Res (2003) 12: 283-290) despite all the evidence (some summarized above) that it should have profound effects. One possible explanation is that there are compensatory effects in the constitutive knock-out animals that obscure the effects of deletion of the A1 receptor. Thus Bjorness et al. conditionally deleted the A1 receptor in brain by using cre-lox mediated recombination to excise a floxed A1 receptor under the control of CAM kinase II driven cre expression. This approach reveals an interesting sleep phenotype. Under control conditions (no sleep restriction), the duration of sleep was unaffected by this targeted deletion of A1 receptors but there was significantly less SWA during slow wave sleep. Bjorness et al. then used acute and chronic sleep deprivation paradigms to study the effects of targeted deletion of A1 receptors. Following acute sleep deprivation, A1 receptor deletion reduced the amount of rebound SWA selectively during slow wave sleep. Under the chronic model of sleep restriction, the effects of A1 deletion on SWA were even greater. Thus, the A1 receptor seems to be particularly important for the rebound in SWA that follows sleep deprivation. This set the stage for these authors to test how this phenotype-restriction of rebound in SWA following sleep deprivation - impacted on memory. They chose a test of working memory. The mouse was allowed to run around a radial arm maze collecting chocolates - revisiting an arm previously raided for chocolate and thus empty was considered a defect of working memory, and the proportion of revisits was taken as a score of working memory. The floxed A1 receptor control mice were able to perform the working memory task during chronic sleep restriction as well as under baseline conditions and when allowed unrestricted sleep. By contrast, the mice with targeted deletion of the A1 receptor performed markedly less well under conditions of sleep restriction. Thus, the authors conclude that SWA does have an important restorative effect and it, rather than the length of slow wave sleep per se, is important for restoration of cognitive function.

How do we link these two papers, which study adenosinergic modulation of sleep and memory by different methods and paradigms? This is an important ongoing task but a recent paper shows that astrocytes do indeed contribute to the development of synchronized cortical oscillations (Fellin T. et al., Proc Natl Acad Sci USA (2009) doi: 10.1073/pnas.0906419106).

\begin{abstract}
About the Author
Nicholas Dale is the Ted Pridgeon Professor of Neuroscience at the University of Warwick. His research interests encompass: 1) the study of purinergic signalling in a variety of contexts such as synaptic transmission, sleep and metabolic stress; 2) the mechanisms of $\mathrm{CO}_{2}$-sensitivity in the brain; and 3) the development of biosensor technologies to study the release of ATP, adenosine and a variety of other transmitters and neuroactive substances such as glutamate, D-serine and lactate.
\end{abstract}

